\title{
Exploring Steinitz-Rademacher polyhedra: a challenge for automated reasoning tools
}

\author{
Jesse Alama* \\ Center for Artificial Intelligence \\ Department of Computer Science, Faculty of Science and Technology \\ New University of Lisbon. Portugal \\ j.alamåfct.unl.pt
}

\begin{abstract}
This note reports on some experiments, using a handful of standard automated reasoning tools, for exploring Steinitz-Rademacher polyhedra, which are models of a certain first-order theory of incidence structures. This theory and its models, even simple ones, presents significant, geometrically fascinating challenges for automated reasoning tools are.
\end{abstract}

\section{Introduction}

This note reports on some experiments, using a handful of standard automated reasoning tools, for exploring a certain first-order theory of three-dimensional polyhedra. Polyhedra are understood here as combinatorial objects, rather than as, say, certain kinds of structures in $\mathbf{R}^{3}$.

Specifically, the polyhedra considered here are Steinitz-Rademacher polyhedra (they will be defined in Section 2). As first-order structures, these polyhedra are directed graphs with three sortsvertices, edges, and faces—-satisfying some intuitive geometric principles shared by "everyday" threedimensional polyhedra.

Restricting ourselves to first-order logic makes it possible to take advantage of automated reasoning tools that work well for FOL, but our restriction comes with a price: many interesting features of graphs, such as connectivity or the property of satisfying Euler's formula, for example, cannot be expressed in FOL. ${ }^{1}$ Nonetheless, it is not clear that FOL's lack of expressive power precludes the possibility of learning something about polyhedra. We believe that the preliminary results discussed here do have interesting mathematical content.

An investigation of polyhedra with automated reasoners is valuable for two domains. First, the investigation is valuable for mathematics, because automated reasoners offer the possibility, in certain contexts, of a more objective investigation than one carried out by entirely by humans, who are prone to make subtle flaws when reasoning about space. Second, the investigation is valuable for automated reasoning, because working with polyhedra—even small ones—naturally leads to challenging problems, as we shall see.

There do not appear to be many explorations of polyhedra using automated reasoning tools. Much has been done on enumerating polyhedra (or related combinatorial structures, such as planar graphs) using mathematical rather than logical techniques; one such system is the highly efficient plantri [1]). Within the realm of automated reasoning, L. Schewe has used SAT solvers to investigate realizability of abstract simplicial complexes [6].

\footnotetext{
${ }^{*}$ Partially supported by the ESF research project Dialogical Foundations of Semantics within the ESF Eurocores program LogICCC (funded by the Portuguese Science Foundation, FCT LogICCC/0001/2007).

${ }^{1}$ FOL's failure to express these and other properties of graphs holds even when one restricts attention to finite structures; such results can be shown using Ehrenfeucht-Fraïssé games [4].
} 


\section{Steinitz-Rademacher polyhedra}

E. Steinitz asked [7]: when can a combinatorially given polyhedron-a collection of abstract vertices, edges, and faces, with an incidence relation among these polytopes-be realized as a three-dimensional convex polyhedron in $\mathbf{R}^{3} ?^{2}$ As part of his initial investigation Steinitz formulated a basic theory of combinatorial polyhedra that forms the basis for our investigation as well.

First, we specify an unsorted first-order signature:

Definition 1. Let $\pi$ be the first-order signature (with equality) containing three unary predicates $V$ (for "vertex"), E (for "edge"), and F (for "face"), and one binary relation I (for incidence).

The signature $\pi$ provides a rudimentary language for talking about three-dimensional polyhedra. Alternatively, one can view $\pi$-structures simply as directed graphs whose nodes can be painted with one of three "colors" $V, E$, and $F$.

Definition 2. The theory SR of Steinitz-Rademacher polyhedra consists of the statements:

- there are vertices, edges, and faces;

- every element is a vertex, edge, or a face;

- I is symmetric;

- no two vertices are incident, and the same goes for edges and faces;

- if $V(v), E(e), F(f), I(v, e)$ and $I(e, f)$, then $I(v, f)$;
- every edge is incident with exactly two vertices;

- every edge is incident with exactly two faces;

- $V(v), F(f)$ and $I(v, f)$ imply that there are exactly two edges incident with both $v$ and $f$; and

- every vertex and every face is incident with at least one other element.

These conditions are expressible as first-order $\pi$-sentences.

Definition 3. A Steinitz-Rademacher polyhedron (or SR-polyhedron) is a model of the theory SR.

Some questions that we would like to address about SR-polyhedra, in this note, are:

1. SR is consistent (just think of, say, a tetrahedron). What is the smallest model? $?^{3}$

2. For which natural numbers $k$ is $\mathrm{SR} k$-categorical? ${ }^{4}$

3. How hard is it to "recover" (that is, produce as models of SR) well-known polyhedra (e.g., the platonic solids) as models of SR?

4. Can we discover unusual or unexpected SR-polyhedra?

The rest of the paper takes up these questions.

Question 1 is also one of the first questions raised by Steinitz and Rademacher [7].

\footnotetext{
${ }^{2}$ The answer, known as Steinitz's theorem [3], says that a directed graph $g$ is isomorphic to the 1-skeleton of a real convex three-dimensional polyhedron iff $g$ is planar and three-connected.

${ }^{3}$ There is no largest finite SR-polyhedron. Consider, for example, the sequence $\left\langle P_{n} \mid n \geq 3\right\rangle$ of pyramids, each $P_{n}$ characterized by an $n$-gon $B_{n}$ for its base and a single point "above" the base incident with each of the vertices of $B_{n}$. Each $P_{n}$ is evidently an SR-polyhedron has cardinality $(n+1)+2 n+(n+1)=4 n+2$.

${ }^{4} \mathrm{SR}$ is not $\lambda$-categorical for any infinite cardinal $\lambda$. Consider, for example, the "tessellation" $M_{\lambda}^{3}$ having $\lambda$ vertices, $\lambda$ edges, and $\lambda$ faces, each of which is a triangle that meets three other triangles along its three edges, ad $\lambda$-infinitum; and $M_{\lambda}^{4}$, which is a "tessellation" like $M_{\lambda}^{3}$ except that each face of $M_{\lambda}^{4}$ has four edges rather than three. In $M_{\lambda}^{3}$ the $\pi$-sentence "every face is a triangle" holds, but by construction it fails in $M_{\lambda}^{4}$. Or, continuing the discussion of the previous footnote, consider a tetrahedron and a cube, each of whose edges and faces contains $\lambda$-many vertices (but each having their usual finite number of edges and faces).
} 
Theorem 4. There is a Steinitz-Rademacher polyhedron of cardinality 6, but none of smaller cardinality.

Proof. This result is readily confirmed with the help of a first-order model generation tool (e.g., mace4 [5] or paradox/equinox [2]). A refutational theorem prover can then show that SR, extended with an axiom saying that there are at most five elements, is inconsistent.

Figure 1 illustrates this smallest SR-polyhedron, $M_{6}$, with two vertices, two edges, and two faces; the two edges are the upper and lower semicircular arcs, and the two faces are the inside and outside of the circle. $M_{6}$ has the curious feature that every vertex is incident with every edge and with every face.

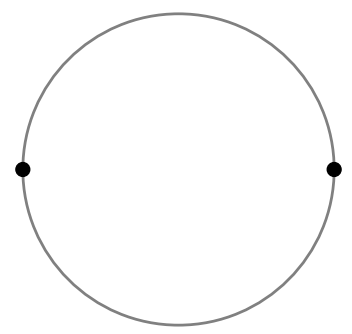

Figure 1: $M_{6}$ : the smallest SR-polyhedron, with six elements: two vertices, two edges, two faces

Depending on one's view about polyhedra, $M_{6}$ might be a positive solution to Question 4; see Section 3

Theorem 5. Up to isomorphism, $M_{6}$ is the only SR-polyhedron of cardinality six.

Proof. Each of the 28 triples of natural numbers $\left(N_{0}, N_{1}, N_{2}\right)$ that sum to 6 gives rise to an extension $\mathrm{SR}_{N_{0}, N_{1}, N_{2}}$ of SR obtained by adding axioms saying that there are exactly $N_{0}$ vertices, $N_{1}$ edges, and $N_{2}$ faces. All but one of these 28 theories-namely, $\mathbf{S R}_{2,2,2}$ - are inconsistent; this can be shown by applying a standard refutational theorem prover to the 27 theories different from $\mathrm{SR}_{2,2,2}$.

To show that $M_{6}$ is, up to isomorphism, the only SR-polyhedron with exactly 2 vertices, 2 edges, and 2 faces, consider the extension of SR by the formula

$$
\varphi:=\exists x_{0}, \ldots, x_{5}\left[\begin{array}{c}
V\left(x_{0}\right) \wedge V\left(x_{1}\right) \wedge x_{0} \neq x_{1} \wedge \forall x\left(V(x) \rightarrow\left(x=x_{0} \vee x=x_{1}\right)\right) \\
\wedge\left(x_{2}\right) \wedge E\left(x_{3}\right) \wedge x_{2} \neq x_{3} \wedge \forall x\left(E(x) \rightarrow\left(x=x_{2} \vee x=x_{3}\right)\right) \\
\wedge\left(x_{4}\right) \wedge F\left(x_{5}\right) \wedge x_{4} \neq x_{5} \wedge \forall x\left(F(x) \rightarrow\left(x=x_{4} \vee x=x_{5}\right)\right) \\
\wedge \\
\neg\left(I\left(x_{0}, x_{2}\right) \wedge I\left(x_{1}, x_{2}\right) \wedge I\left(x_{0}, x_{3}\right) \wedge I\left(x_{1}, x_{3}\right) \wedge I\left(x_{2}, x_{4}\right) \wedge I\left(x_{3}, x_{4}\right) \wedge I\left(x_{2}, x_{5}\right) \wedge I\left(x_{3}, x_{5}\right)\right)
\end{array}\right]
$$

The first three bundles of conjunctions in the matrix of $\varphi$ express the cardinality of the sets of vertices, edges, and faces. The final conjunction expresses the essential incidence relations holding among the elements of $M_{6}$, when one labels the vertices $x_{0}$ and $x_{1}$, the edges $x_{2}$ and $x_{3}$, and the faces $x_{4}$ and $x_{5}$; it is negated because we are trying to find a model that is unlike $M_{6}$. One then shows, with, e.g., mace4, that $\mathrm{SR} \cup\{\varphi\}$ is unsatisfiable.

\section{Theorem 6. There are at least two SR-polyhedron of cardinality 8.}

One such cardinality 8 SR-polyhedron is depicted in Figure 2 The two vertices are clear; the three edges are the upper and lower semicircles, plus the straight line segment joining the two vertices. The three faces are: the exterior of the circle, incident with the upper and lower semicircular arcs; and the 


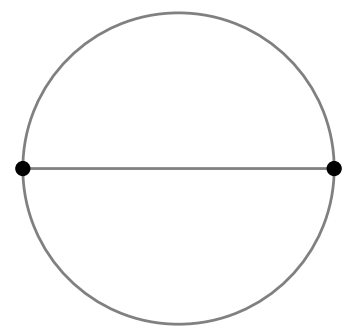

Figure 2: An SR-polyhedron with eight elements: two vertices, three edges, three faces.

two semicircles, each incident with one of the semicircular arcs and both incident with the straight line segment. Another is its dual, $M_{8}^{d}$, which is obtained by exchanging the vertices and faces of $M_{8}$ (notice that $\mathrm{SR}$ is invariant under this exchange).

Returning to Question 4, as with, $M_{6}$, the SR-polyhedron $M_{8}$ and its dual $M_{8}^{d}$ could be regarded as an unusual or unexpected model of SR. Although SR does admit curious "polyhedra", it should be clear that one can take nearly any familiar polyhedron, such as the platonic solids, as SR-polyhedra.

\begin{tabular}{l|r|r|r|r} 
Solid & Num. Vertices & Num. Edges & Num. Faces & Cardinality \\
\hline Tetrahedron & 4 & 6 & 4 & 14 \\
Cube & 8 & 12 & 6 & 26 \\
Octahedron & 6 & 12 & 8 & 26 \\
Dodecahedron & 20 & 30 & 12 & 62 \\
Icosahedron & 12 & 30 & 20 & 62
\end{tabular}

Table 1: Cardinal numbers for the platonic solids

Notice that, since they are duals, the cube and the octahedron, as well as the dodecahedron and the icosahedron, show that SR is neither 26- nor 62-categorical. We now have the modest beginnings of an answer to Question 2: for $k=6$ we have that SR is $k$-categorical, and for $k=8,26$, and 62 we know that $\mathrm{SR}$ is not $k$-categorical, by duality.

Although the tetrahedron can be recovered as an SR-polyhedron (by, e.g., paradox), the remaining platonic solids seem to lie tantalizingly beyond the scope of current automated reasoning tools: a very large amount of time indeed seems to be required to automatically generate these solids. It would be interesting to determine whether the cube and its dual the icosahedron are the only SR-polyhedra of cardinality 26.

For lack of space the investigation has to be cut short here.

\section{Future work}

The range of Steinitz-Rademacher polyhedra is arguably too large: if the tetrahedron is the "simplest" three-dimensional polyhedron, then the six-element SR-polyhedron $M_{6}$ in Figure 2 and other SR-polyhedra of cardinality less than that of the tetrahedron, such as $M_{8}$ in Figure 2 show that SR alone lacks sufficient geometric content and needs to be extended by principles that rule out such models. Extensionality is a natural candidate. $M_{6}$, for example, has two distinct vertices that share the same edges and faces, two distinct edges that share the same two vertices and faces, and two distinct faces that share the same vertices and edges. The eight-element model $M_{8}$ is similar. Intuitively, the incidence 
relation between polytopes is extensional: if two polytopes $p$ and $q$ are incident with the same set of polytopes, then $p=q$.

Adding extensionality to $\mathrm{SR}$ yields a new theory $\mathrm{SR}_{\mathrm{ext}}$ of extensional $\mathrm{SR}$-polyhedra. paradox can recover the tetrahedron as the smallest model of $S_{R_{\text {ext }}}$, thereby answering Question 1 and part of Question 3 for the new theory. However, extensionality raises a high computational hurdle. Concerning Questions 2 and 3, the search for models of $\mathrm{SR}_{\text {ext }}$ has so far not been successful beyond the aforementioned recovery of the tetrahedron. Question 4, about $k$-categoricity, is an even greater challenge for $\mathrm{SR}_{\mathrm{ext}}$ than it was for SR and is more intriguing because its models are more geometrically intuitive.

The signature $\pi$ of SR-polyhedra is unsorted: $V, E, F$, and $I$ are relations that could hold for arbitrary elements in a $\pi$-structure. It is likely that tools for sorted FOL, such as SPASS [8], would be more effective than the unsorted tools used so far. Constraint techniques for model generation, such as those behind sem [9], ought also to be evaluated.

\section{Conclusion}

Combinatorial polyhedra abstract away from positions in space and regard polyhedra as incidence structures. Steinitz-Rademacher polyhedra are one such kind of polyhedra axiomatized by an intuitive firstorder theory. We have proposed a handful of basic questions about these polyhedra and shown that automated reasoners can tackle some of them with verve, though others remain only partially answered. Even small Steinitz-Rademacher polyhedra present significant challenges for automated reasoners; dealing with larger ones will likely require new techniques. We thus urge combinatorial polyhedra as a tantalizingly fertile source of challenging automated reasoning problems.

\section{References}

[1] G. Brinkmann and B. D. McKay. Fast generation of planar graphs. MATCH Comm. in Comp. Chem., 58:323357, 2003.

[2] K. Claessen and N. Sorensson. New Techniques that Improve MACE-style Finite Model Finding. In P. Baumgartner and C. Fermueller, editors, Proc. of the CADE-19 Workshop: Model Computation: Principles, Algorithms, Applications, 2003.

[3] B. Grünbaum. Graphs of polyhedra; polyhedra as graphs. Discrete Mathematics, 307:445-463, 2007.

[4] L. Libkin. Elements of Finite Model Theory. Texts in Theoretical Computer Science. Springer, 2004.

[5] W. McCune. Prover9 and Mace4. http://www.cs.unm.edu/ mccune/prover9/

[6] L. Schewe. Generation of oriented matroids using satisfiability solvers. In A. Iglesias and N. Takayama, editors, Proc. of the 2nd Int. Conf. on Math. Soft., volume 4151 of Lecture Notes in Computer Science. Springer, 2006.

[7] E. Steinitz and H. Rademacher. Vorlesungen über die Theorie der Polyeder unter Einschluss der Elemente der Topologie. Springer, 1976. Reprint of the original 1934 edition.

[8] C. Weidenbach, D. Dimova, A. Fietzke, R. Kumar, M. Suda, and P. Wischnewski. SPASS version 3.5. In R. Schmidt, editor, Automated Deduction: CADE 22, volume 5663 of Lecture Notes in Computer Science, pages 140-145. Springer, 2009.

[9] J. Zhang and H. Zhang. SEM: A system for enumerating models. In C. P. Mellish, editor, Proceedings of the Fourteenth International Joint Conference on Artificial Intelligence, pages 298-303, 1995. 